\title{
Exploring lag phase and growth initiation of a yeast culture by means of an Individual-based Model
}

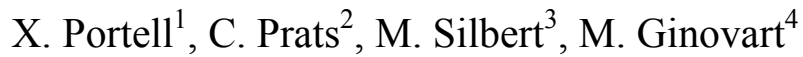 \\ ${ }^{1}$ Department of Agri-Food Engineering and Biotechnology, Technical University of Catalonia, Barcelona, Spain \\ (xavier.portell@upc.edu) \\ ${ }^{2}$ Department of Physics and Nuclear Engineering, Technical University of Catalonia, Barcelona, Spain \\ (clara.prats@upc.edu) \\ ${ }^{3}$ Institute of Food Research, Norwich Research Park Colney, Norwich, UK (moises.silbert@bbsrc.ac.uk) \\ ${ }^{4}$ Department of Applied Mathematics III, Technical University of Catalonia, Barcelona, Spain \\ (marta.ginovart@upc.edu)
}

\begin{abstract}
Yeast ageing and inoculum size are factors that affect successive industrial fermentation, particularly in those processes that reuse the yeast cells. The aim of the work is to explore the effects of inocula size and aging on the dynamics of yeast population. However, only individual-based modelling (IbM) makes possible studies of small, well characterized, microbial inocula. Here we have made use of INDISIM-YEAST to carry out these studies. Several simulations were performed to analyze inoculum size and their different genealogical ages on the lag phase, first division time and specific growth rate. Shortest lag phase and time to the first division where obtained with largest inocula and with youngest inoculated parent cells.
\end{abstract}

Keywords: Individual-based model, lag phase, growth initiation, yeast inocula

\section{Introduction}

In industrial applications yeast is usually propagated in a number of steps before being inoculated into the final fermentation medium. The inoculated culture is not often well defined in spite of the fact that the physiological condition of the yeast cells may greatly affect the duration and outcome of the fermentation (Walker 1998). For instance, the production of beer reuses yeast cropped at the end of fermentation in subsequent fermentation, so yeast is maintained and reused a number of times, a process called 'serial repitching'. When yeast cells are inoculated into a fresh growth medium, these enter a brief lag phase where they are biochemically active but they still do not divide. After this lag phase, cells go into their cell cycle and start dividing. We are concerned here with yeast budding reproduction, which leads to scar formation. The genealogical age of yeast cells and the small size of daughter cells in front of older cells are two individual characteristics that influence the evolution of a culture at the beginning of its development.

The microbial lag phase has usually been investigated with continuous population models using rather high inoculum levels. When microbial growth is considered starting from a few cells, the study of this evolution demands an individual-based approach. The two approaches, however, can converge to similar results as the size of the population increases (GómezMourelo and Ginovart, 2009).

Unlike continuous models, IbM is a bottom-up approach. Of those available (Hellweger and Bucci 2009) we have used INDISIM, the simulator developed by our group ( Ginovart et al. 2002, Ferrer et al. 2008), and which has already been used to study different features of the bacterial lag phase providing an ample pool of interesting results (Prats et al. 2006, 2008). INDISIM-YEAST constitutes the adaptation of INDISIM to study the specific characteristics of the yeast cell cycle to take care of yeast populations growing in liquid media (Ginovart et al. 2007, Ginovart and Cañadas 2008). This simulator has recently been used to attempt to study some aspects of the influence of cell ageing on the fermentation processes (Ginovart et al. 2009). 
The aim of this contribution is to explore the effects of specific characteristics of the initial inocula on the dynamics of a yeast culture in liquid medium during the lag phase and the first stages of growth using the individual-based simulator INDISIM-YEAST.

\section{Material and methods}

For each yeast cell, INDISIM-YEAST implements a set of rules for uptake and metabolism of nutrient particles, excretion of end products, budding reproduction and viability. The yeast population is made up of a set of cells with individual variables defining them (position in the spatial domain, biomass, genealogical age as the number of bud scars on the cellular membrane, reproduction phase in the cellular cycle where it is the unbudded or budding phase, "start mass" or mass required to change from the unbudded to budding phase, minimum growth for the budding phase, minimum time to complete the budding phase and survival time without satisfying its metabolic requirements). The description of the principal concepts of this yeast cell modelling plus the different elements to assemble the structure system for the virtual process of glucose fermentation can be found in the works of Ginovart et al. (2007) and Ginovart and Cañadas (2008).

The main simulation result shown in the present study is the temporal evolution of the yeast population that grows from an inoculum, which is completely characterized. Special attention is focused during the first stages of its development until the population reaches the exponential phase. There is no nutrient limitation on the initial conditions of the simulated culture.

Two parameters are used to characterize the outcome of a given culture growth during the first stages. The first is the classic lag parameter, defined at the population level of description and calculated through its geometrical definition. When the population reaches its maximum and enters the stationary phase, a logarithmic regression of an upper interval in the exponential growth is performed to obtain the straight line $\operatorname{Ln} N=\mu t+\mathrm{b}$ and the maximum growth rate $\mu$ (Figure 1). The intersection of the prolongation of this straight line with the $\mathrm{LnN}_{0}$ line gives the lag time $\lambda$, where $\lambda=\left(\mathrm{LnN}_{0}-\mathrm{b}\right) / \mu$. In order to characterise much better the initial steps of the yeast population at an individual level of description, a second parameter is also considered, the time when the First microbial Division $\left(t_{F D}\right)$ takes place or the first budding reproduction appears (Figure 1).

Several simulations to evaluate the above parameters have been carried out with yeast cells of different genealogical ages making up the inocula (namely samples that include daughter cells or virgin cells with 0 scars and/or parent cells with $1,2,3, \ldots .7$ or 8 scars on their membranes), and with inocula of different sizes (the number of yeast cells to begin the evolution is set from 1 cell to 1000 cells).
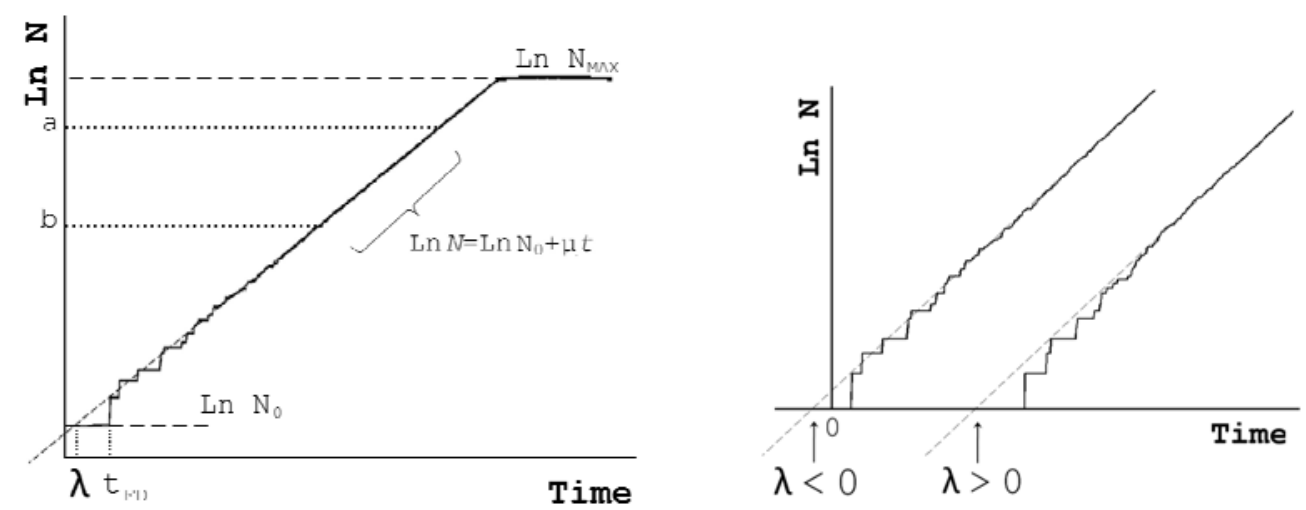

Figure 1: Calculation of the following parameters: lag time, first division time and maximum growth rate (exponential phase). Left: geometrical method to obtain $\lambda$ and $\mu$, where $\mathrm{a}=\mathrm{LnN}_{0}+0.85\left(\mathrm{LnN}_{\mathrm{MAX}}-\mathrm{LnN}_{0}\right)$ and $\mathrm{b}=\mathrm{LnN}_{0}+0.60\left(\mathrm{LnN}_{\mathrm{MAX}}-\mathrm{LnN}_{0}\right)$. Right: two different simulations to show how negative and positive lag times can be achieved. 


\section{Results and discussion}

We present the results of two series of simulations carried out using INDISIM-YEAST with different virtual inocula. From a heterogeneous population of yeast cells successive inocula have been obtained which, in turn became the initial seeds to perform those simulations. The first series (with 50 runs for each case) is designed to evaluate the effect of the genealogical age of the inoculum, which is made up of a unique yeast cell, on the parameters $\lambda, t_{\mathrm{FD}}$ and $\mu$ of the growth curve (Figure 2). The second, also with 50 simulations in each case, studies yeast growth from different size inocula randomly taken from the aforementioned population (Figure 3). Low inocula growth curves have high synchronisms during the first stages of the temporal evolution. These synchronisms result in a geometrical effect that deforms the geometrical evaluation of the lag phase (Figure 1). Moreover, the fact that in some cases the lag time is shorter that its corresponding time for the first bud reproduction (Figures 2 and 3) is not consistent with the concept that lag phase desires to represent. For single-cell inocula (Figure 2) both parameters have their minimum value for youngest parent yeast. As the genealogical age of the inoculum increases, $\lambda$ and $t_{\mathrm{FD}}$ became longer. Daughter cells also have longer lags than young parent cells. Regarding the inoculum size, large inocula reach the exponential phase sooner than small inocula do (Figure 3). A third series of simulations has been performed selecting inocula that combine the preceding two factors, size and genealogical ages. That is, different inocula sizes with only virgin cells ( 0 scars), middle-age cells (1-5 scars) or old cells have been chosen to perform sets of 50 runs for each combination (data not shown). All these simulations results show there is an influence of these initial features of the inocula on $\lambda$ and $t_{\mathrm{FD}}$, and only slight discrepancies on $\mu$ are observed.
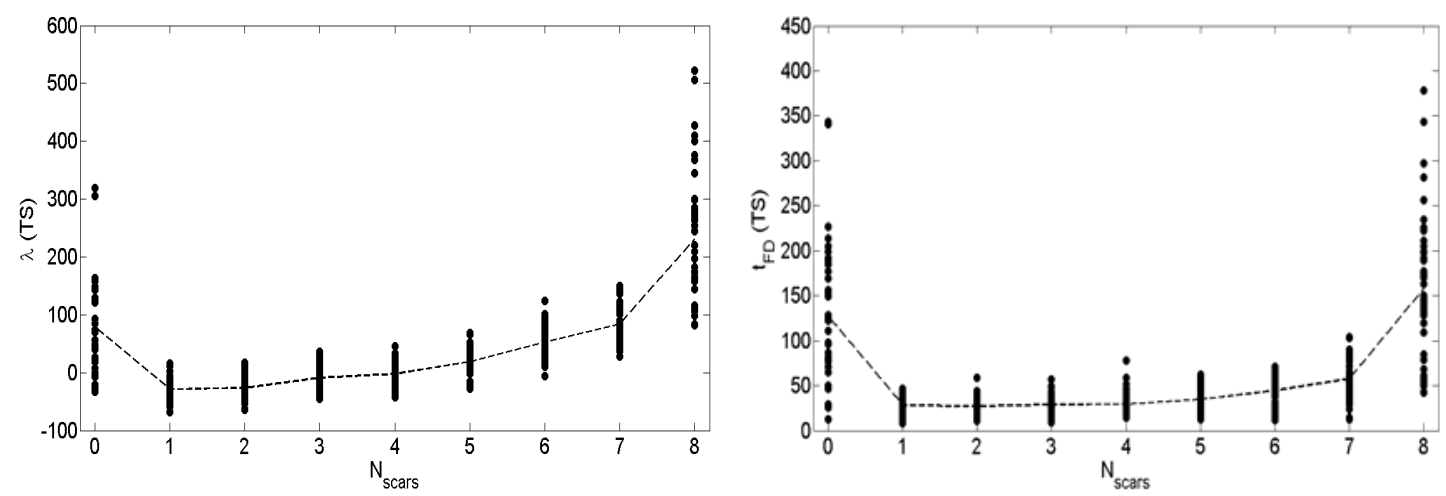

Figure 2: First simulations series (see text). Lag parameter (left) and first division time (right) versus the number of scars of the single-cell inoculum. The dashed line indicates the mean value for each genealogical age.
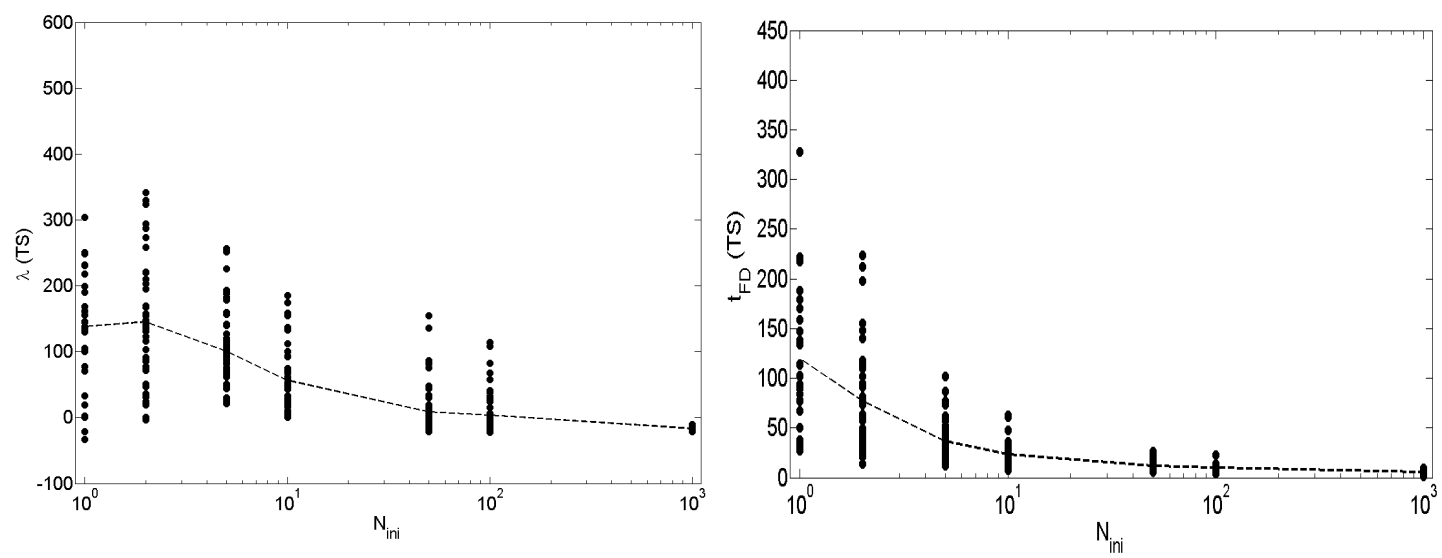

Figure 3: Second simulations series (see text). Lag parameter (left) and first division time (right) versus inoculum size. The dashed line indicates the mean value for each inoculum size. 
Within the stated limitations, this study mimics the industrial production of beer, which reuses yeast cropped at the end of fermentation in subsequent fermentations, so the immediate and long term fermentation performance is conditioned by the characteristics of these reused inocula. Since the yeast Saccharomyces cerevisae has a limited replicative lifespan, each cell within a population is only capable of a finite number of divisions prior to senescence and death (Powell et al., 2000, 2003). Towards the end of fermentation yeast sediments and are collected within the fermenter cone. Sedimentation results in the formation of zones enriched with cells of a particular age. At the end of a fermentation a portion of the yeast is removed from the fermentation vessel for 'serial repitching'. Typically this is the centre-top portion of the yeast crop, theoretically comprising middle-aged and virgin cells (Powell et al., 2003).

\section{Conclusions}

We have shown that the IbM INDISIM-YEAST is capable of distinguishing the differences in the evolution of a population that emerges from a small inocula, whether it started with a single microorganism, a population make up by different genealogical ages, or different sizes. It is particularly useful in the study of small inocula and the initial steps of the population evolution because of the excessive influence of the discrete and asymmetrical nature of yeast division. It is here that it has an edge compared to top-down continuous models, which are useful only when the initial population contains a large number of cells. The tendencies found in the simulations resemble those seen during 'serial repitching' in beer fermentation.

\section{Acknowledgements}

We thank Rosa Carbó for several comments and suggestions. We gratefully acknowledge the financial support received from the Ministry of Education and Science (Plan Nacional I+D+i) through Grant CGL2007-65142/BOS, and the Universitat Politècnica de Catalunya (UPC).

\section{References}

Ferrer J., Prats C. and López D. (2008) Individual-based modeling: An essential Tool for Microbiology. Journal of Biological Physics 34 (1-2), 19-37.

Ginovart M., López D. and Valls J. (2002) INDISIM, and individual based discrete simulation model to study bacterial cultures. Journal of Theoretical Biology 214 (2), 305-319.

Ginovart M., Xifré J., López D. and Silbert M.: INDISIM-YEAST, an individual-based model to study yeast population in batch cultures. In: Méndez-Vilas A (ed) Communicating current research and educational topics and trends in applied microbiology, Microbiology book series n ${ }^{\circ} 1$ Vol. 1, Formatex, Badajoz, 2007, 401-409.

Ginovart M. and Cañadas, J.C. (2008) INDISIM-YEAST: an individual-based simulator on a website for experimenting and investigating diverse dynamics of yeast populations in liquid media. Journal of Industrial Microbiology and Biotechnology 35 (11), 1359-1366.

Ginovart M., Portell X., Silbert M. (2009) Repitching of yeast in beer fermentations: individual-based model simulations. In I. Troch and F. Breitenecker (Eds.), Proceedings MATHMOD 09 Vienna, in press, ARGESIM Report no. 35 (ISBN 978-3-901608-35-3) [MATHMOD Vienna-Vienna International Conference on Mathematical Modelling, Vienna (Austria), February 11-13, 2009].

Gómez-Mourelo P. and Ginovart M. (2009) The differential equation counterpart of an individual-based model for yeast population growth. Computers \& Mathematics with Applications, in press.

Hellweger F.L. and Bucci V. (2009) A bunch of tiny individuals - Individual-based modeling for microbes. Ecological Modelling 220 (1), 8-22.

Powell C.D., Van Zandyke S.M., Quain D.E. and Smart K.A. (2000) Replicative ageing and senescence in Saccharomyces cerevisiae and the impact on brewing fermentations. Microbiology 146 (5), 1023-1034.

Powell C.D., Quain D.E. and Smart K.A. (2003) The impact of brewing yeast cell age on fermentation performance, attenuation and flocculation. FEMS Yeast Research 3 (2), 149-157.

Prats C., López D., Giró A., Ferrer J. and Valls J. (2006) Individual-based modelling of bacterial cultures to study the microscopic causes of the lag phase. Journal of Theoretical Biology 241 (4), 939-953.

Prats C., Giró A., Ferrer J., López D., Vives-Rego J. (2008) Analysis and IbM simulation of the stages in bacterial lag phase: Basis for an updated definition. Journal of Theoretical Biology 22 (1), 56-68.

Walker G.M. (1998) Yeast physiology and biotechnology, JohnWiley \& Sons, Chichester, UK, 350 pp. (ISBN 0471-96446-8). 